\title{
A Lyapunov functional for a system with both lumped and distributed delay
}

\author{
JOZEF DUDA
}

\begin{abstract}
In the paper construction of a Lyapunov functional for time delay system with both lumped and distributed delay is presented. The Lyapunov functional is determined by means of the Lyapunov matrix. The method of determination of the Lyapunov matrix for time delay system with both lumped and distributed delay is presented. It is given the example illustrating the method.
\end{abstract}

Key words: time-delay system, Lyapunov matrix, Lyapunov functional.

\section{Introduction}

The Lyapunov functionals are used to test the stability of the systems. For example Fridman [11] introduced the Lyapunov-Krasovskii functional for examination the stability of the linear retarded and neutral type systems with discrete and distributed delays, which were based on equivalent descriptor form of the original system and obtained delay-dependent and delay-independent conditions in terms of linear matrix inequality (LMI). Ivanescu et al. [23] proceeded with the delay-depended stability analysis for the linear neutral systems, constructed the Lyapunov functional and derived sufficient delaydependent conditions in terms of linear matrix inequalities (LMIs). Han [17] obtained a delay-dependent stability criterion for the neutral systems with a time-varying discrete delay. This criterion was expressed in the form of LMI and was obtained using the Lyapunov direct method. Han [18] developed the discretized Lyapunov functional approach to investigate the stability of linear neutral systems with mixed neutral and discrete delays. The stability criteria, which are applicable to linear neutral systems with both small and non-small discrete delays, are formulated in the form of LMIs. Han [19] studied the stability problem of linear time delay systems, both retarded and neutral types, using the discrete delay $\mathrm{N}$-decomposition approach to derive some new more general discrete delay dependent stability criteria. Han [20] employed the delay-decomposition approach

The Author is with Department of Automatics and Biomedical Engineering, AGH University of Science and Technology, Krakow, Poland. E-mail: jduda@agh.edu.pl

The author wishes to thank the editors and the reviewers for their suggestions, which have improved the quality of the paper.

Received 1.03.2017. Revised 13.9.2017. 
to derive some improved stability criteria for linear neutral systems and to deduce some sufficient conditions for the existence of a Lyapunov functional for a system with k-noncommensurate neutral time delays of a delayed state feedback controller, which ensure asymptotic stability and a prescribed $\mathrm{H} 1$ performance level of the corresponding closedloop system. Gu and Liu [16] investigated the stability of coupled differential-functional equations using the discretized Lyapunov functional method and delivered the stability condition in the form of LMI, suitable for numerical computation.

The Lyapunov functionals are also used in calculation of the robustness bounds for uncertain time delay systems. For illustration Kharitonov and Zhabko [30] proposed a procedure of construction of the quadratic functionals for the linear retarded type delay systems which could be used for the robust stability analysis of time delay systems. This functional was expressed by means of Lyapunov matrix, which depended on the fundamental matrix of a time delay system. Kharitonov [24] extended some basic results obtained for the case of retarded type time delay systems to the case of neutral type time delay systems, and in [25] to the neutral type time delay systems with a discrete and distributed delay. Han [21] investigated the robust stability of uncertain neutral systems with discrete and distributed delays, which has been based on the descriptor model transformation and the decomposition technique, and formulated the stability criteria in the form of LMIs. Han [22] considered the stability for the linear neutral systems with norm-bounded uncertainties in all system matrices and derived a new delay-dependent stability criterion. Neither model transformation nor bounding technique for cross terms is involved through derivation of the stability criterion.

The Lyapunov functionals are also used in computation of the exponential estimates for the solutions of the time delay systems. For instance Kharitonov and Hinrichsen [28] used the Lyapunov matrix to derive exponential estimates for the solutions of exponentially stable time delay systems. Kharitonov and Plischke [29] formulated the necessary and sufficient conditions for the existence and uniqueness of the delay Lyapunov matrix for the case of a retarded system with one delay. The numerical scheme for construction of the Lyapunov functionals has been proposed by $\mathrm{Gu}$ [15].This method starts with the discretisation of a Lyapunov functional. The scheme is based on LMI techniques.

There are papers whose regard the quadratic Lyapunov functionals such that their coefficients are given by the analytical formulas. One constructs the Lyapunov functionals for the system with a time delay with a given time derivative. For the first time such Lyapunov functional was introduced by Repin [32] for the case of retarded time delay linear systems with one delay. Repin [32] delivered also the procedure for determination of coefficients of the functional. Duda [3] presented that method of determining of the Lyapunov functional for a linear dynamical system with two lumped retarded type time delays in the general case with non- commensurate delays and presented a special case with commensurate delays in which the Lyapunov functional could be determined by solving of the ordinary differential equations set. Duda [4] presented also the method of determining of the Lyapunov functional for a neutral system with k-non-commensurate delays and in [9] for a linear system with both lumped and distributed delay, and in [5] for a system with a time-varying delay. 
The Lyapunov quadratic functionals are also used to calculation of a value of a quadratic performance index of quality in the process of the parametric optimization for the time delay systems. One constructs a Lyapunov functional for the system with a time delay with a given time derivative whose is equal to the negatively defined quadratic form of a system state. The value of that functional at the initial state of the time delay system is equal to the value of a quadratic performance index of quality. In last years a method of determination of a Lyapunov functional by means of the Lyapunov matrix is very popular. Duda used this method in parametric optimization problem for a system with one retarded type time delay and a P-controller [6], for a neutral system with one delay and a P-controller [7], for a retarded type time delay system with two delays and P-controller [8] and for a neutral system with two delays and P-controller [10].

There is another method to achieve a value of a quadratic performance index presented by Górecki and Popek [14] which bases on a characteristic quasi-polynomial. Górecki and Białas published two articles [2,12] whose concern relations between roots of the transcendental equations and their coefficients. These results are helpful in the stability analysis of the time delay systems.

In the paper a Lyapunov functional for time delay system with both lumped and distributed delay is determined by means of the Lyapunov matrix. The method of determination of the Lyapunov matrix for time delay system with both lumped and distributed delay is presented. To the best of author's knowledge, such extension has not been reported in the literature. There is also presented an example illustrating that method.

\section{Mathematical model of a time delay system with both lumped and distributed delay}

Let us consider a time-delay system which dynamics is described by equation

$$
\left\{\begin{array}{l}
\frac{d x(t)}{d t}=A x(t)+B x(t-h)+\int_{-h}^{0} C x(t+\varsigma) d \varsigma \\
x(\theta)=\varphi(\theta)
\end{array}\right.
$$

for $t \geqslant 0, \theta \in[-2 h, 0]$, where $x(t) \in \mathbb{R}^{n}, A, B, C \in \mathbb{R}^{n \times n}, h \geqslant 0$, function $\varphi \in$ $P C\left([-2 h, 0], \mathbb{R}^{n}\right)$ - the space of piece-wise continuous vector valued functions defined on the segment $[-2 h, 0]$ with the uniform norm $\|\varphi\|_{P C}=\sup _{\theta \in[-2 h, 0]}\|\varphi(\theta)\|$.

We introduce a new variable

$$
y(t)=\int_{-h}^{0} x(t+\varsigma) d \varsigma
$$

and compute its time derivative taking into account the relationship 


$$
\begin{gathered}
\frac{d x(t+\varsigma)}{d t}=\frac{d x(t+\varsigma)}{d \varsigma} \\
\frac{d y(t)}{d t}=\int_{-h}^{0} \frac{d}{d t} x(t+\varsigma) d \varsigma=\int_{-h}^{0} \frac{d}{d \varsigma} x(t+\varsigma) d \varsigma=x(t)-x(t-h) .
\end{gathered}
$$

There holds a relationship

$$
y(\sigma)=\int_{-h}^{0} x(\sigma+\theta) d \theta=\int_{-h}^{0} \varphi(\sigma+\theta) d \theta=\psi(\sigma)
$$

for $\sigma \in[-h, 0]$. We obtained the set of equations

$$
\left\{\begin{array}{l}
{\left[\begin{array}{c}
\frac{d x(t)}{d t} \\
\frac{d y(t)}{d t}
\end{array}\right]=\left[\begin{array}{ll}
A & C \\
I & 0
\end{array}\right]\left[\begin{array}{l}
x(t) \\
y(t)
\end{array}\right]+\left[\begin{array}{cc}
B & 0 \\
-I & 0
\end{array}\right]\left[\begin{array}{l}
x(t-h) \\
y(t-h)
\end{array}\right]} \\
{\left[\begin{array}{l}
x(\theta) \\
y(\theta)
\end{array}\right]=\left[\begin{array}{l}
\varphi(\theta) \\
\psi(\theta)
\end{array}\right]}
\end{array}\right.
$$

for $t \geqslant 0, \theta \in[-h, 0]$. We introduce

$$
\begin{aligned}
& z(t)=\left[\begin{array}{l}
x(t) \\
y(t)
\end{array}\right] \\
& A_{0}=\left[\begin{array}{ll}
A & C \\
I & 0
\end{array}\right] \\
& A_{1}=\left[\begin{array}{cc}
B & 0 \\
-I & 0
\end{array}\right]
\end{aligned}
$$

and

$$
\phi=\left[\begin{array}{c}
\varphi \\
\psi
\end{array}\right] \in P C\left([-h, 0], \mathbb{R}^{2 n}\right) .
$$

The system (6) takes the form

$$
\left\{\begin{aligned}
\frac{d z(t)}{d t} & =A_{0} z(t)+A_{1} z(t-h) \\
z(\theta) & =\phi(\theta)
\end{aligned}\right.
$$

for $t \geqslant 0$ and $\theta \in[-h, 0]$, where $A_{0}, A_{1} \in \mathbb{R}^{2 n \times 2 n}$ and $\phi \in P C\left([-h, 0], \mathbb{R}^{2 n}\right), 0 \leqslant h \in \mathbb{R}$. $z(t, \phi)$ is the solution of system (11) with the initial function $\phi$.

The theorems of existence, continuous dependence and uniqueness of solutions of system (11) are given in [13]. 
Definition $2 K(t)$ is the fundamental matrix of system (11) if it satisfies the matrix equation

$$
\frac{d}{d t} K(t)=A_{0} K(t)+A_{1} K(t-h)
$$

for $t \geqslant 0$ and the following initial condition $K(0)=I_{n \times n}$ and $K(t)=0_{n \times n}$ for $t<0$ where $I_{n \times n}$ is the identity $n \times n$ matrix and $0_{n \times n}$ is the zero $n \times n$ matrix.

Theorem 2 (Bellman \& Cooke [1]). Let $K(t)$ be the fundamental matrix of system (11), then for $t \geqslant 0$

$$
z(t, \phi)=K(t) \phi(0)+\int_{-h}^{0} K(t-h-\theta) A_{1} \phi(\theta) d \theta
$$

Definition 3 The function $z_{t}(\phi):[-h, 0] \rightarrow \mathbb{R}^{2 n}$ is called a shifted restriction of $z(\cdot, \phi)$ to an interval $[t-h, t]$ and is defined by a formula

$$
z_{t}(\phi)(\theta):=z(t+\theta, \phi)
$$

for $t \geqslant 0$ and $\theta \in[-h, 0]$.

Definition 4 The trivial solution of (11) is said to be stable iffor any $\varepsilon>0$ there is $\delta>0$ such that

$$
\|\phi\|_{P C} \leqslant \delta \Rightarrow\|z(t, \phi)\| \leqslant \varepsilon
$$

for every $t \geqslant 0$.

Definition 5 The trivial solution of (11) is said to be asymptotically stable if it is stable and $z(t, \phi) \rightarrow 0$ as $t \rightarrow \infty$

Definition 6 The trivial solution of (11) is said to be exponentially stable if there exist $M \geqslant 1$ and $\sigma>0$ such that for every solution $z(t, \phi)$ of the system with initial function $\phi \in P C\left([-h, 0], \mathbb{R}^{2 n}\right)$ the following condition holds

$$
\|z(t, \phi)\| \leqslant M\|\phi\|_{P C} e^{-\sigma t}
$$

for every $t \geqslant 0$.

The controllability of the systems with time delay is presented in [31]. 


\section{A Lyapunov-Krasovskii functional}

Given a symmetric positive definite matrix $W \in \mathbb{R}^{n \times n}$. We are looking for a functional $v$ : $P C\left([-h, 0], \mathbb{R}^{2 n}\right) \rightarrow \mathbb{R}$ such that along the solutions of system (11) the following equality holds

$$
\frac{d}{d t} v\left(z_{t}(\phi)\right)=-z^{T}(t, \phi) W z(t, \phi)
$$

for $t \geqslant 0$, where $z(t, \phi)$ is a solution of system (11), with the initial function $\phi \in$ $P C\left([-h, 0], \mathbb{R}^{2 n}\right)$, given by $(12)$.

We assume that system (11) is asymptotically stable and integrate both side of (14) from zero to infinity. We obtain

$$
v(\phi)=\int_{0}^{\infty} z^{T}(t, \phi) W z(t, \phi) d t .
$$

Taking into account (12) we calculate the integral of the right-hand side of (15)

$$
\begin{aligned}
& \int_{0}^{\infty} z^{T}(t, \phi) W z(t, \phi) d t=\phi^{T}(0) \int_{0}^{\infty} K^{T}(t) W K(t) d t \phi(0)+ \\
& \quad+\int_{-h}^{0} 2 \phi^{T}(0) \int_{0}^{\infty} K^{T}(t) W K(t-h-\theta) d t A_{1} \phi(\theta) d \theta+ \\
& +\int_{-h}^{0} \phi^{T}(\theta) A_{1}^{T} \int_{-h}^{0} \int_{0}^{\infty} K^{T}(t-h-\theta) W K(t-h-\eta) d t A_{1} \phi(\eta) d \eta d \theta .
\end{aligned}
$$

There holds a relation

$$
\begin{gathered}
\int_{0}^{\infty} K^{T}(t-h-\theta) W K(t-h-\eta) d t=\int_{-h-\theta}^{\infty} K^{T}(\varsigma) W K(\varsigma+\theta-\eta) d \varsigma= \\
=\int_{0}^{\infty} K^{T}(\varsigma) W K(\varsigma+\theta-\eta) d \varsigma .
\end{gathered}
$$

We introduce a Lyapunov matrix

$$
U(\xi)=\int_{0}^{\infty} K^{T}(t) W K(t+\xi) d t .
$$

Using the Lyapunov matrix (17) we attain a formula for the functional $v(\phi)$ 


$$
\begin{aligned}
v(\phi)= & \phi^{T}(0) U(0) \phi(0)+2 \phi^{T}(0) \int_{-h}^{0} U(-\theta-h) A_{1} \phi(\theta) d \theta+ \\
& +\int_{-h}^{0} \phi^{T}(\theta) A_{1}^{T} \int_{-h}^{0} U(\theta-\eta) A_{1} \phi(\eta) d \eta d \theta
\end{aligned}
$$

where the Lyapunov matrix $U$ is obtained by solving the set of equations [6]

$$
\begin{gathered}
\frac{d}{d \xi} U(\xi)=U(\xi) A_{0}+U(\xi-h) A_{1} \\
U(-\xi)=U^{T}(\xi) \\
U(0) A_{0}+U(-h) A_{1}+A_{0}^{T} U(0)+A_{1}^{T} U(h)=-W
\end{gathered}
$$

for $\xi \in[0, h]$, where $W$ is a symmetric positive definite matrix. Formula (20) implies

$$
U(\xi-h)=U^{T}(h-\xi)=Z(\xi) .
$$

We compute the derivative of $Z(\xi)$

$$
\frac{d}{d \xi} Z(\xi)=\frac{d}{d \xi} U^{T}(h-\xi)=-A_{0}^{T} U^{T}(h-\xi)-A_{1}^{T} U^{T}(-\xi)=-A_{0}^{T} Z(\xi)-A_{1}^{T} U(\xi) .
$$

We have received the set of ordinary differential equations

$$
\left\{\begin{array}{l}
\frac{d}{d \xi} U(\xi)=U(\xi) A_{0}+Z(\xi) A_{1} \\
\frac{d}{d \xi} Z(\xi)=-A_{0}^{T} Z(\xi)-A_{1}^{T} U(\xi)
\end{array}\right.
$$

for $\xi \in[0, h]$ with initial condition $U(0), Z(0)$. Formula (22) implies

$$
U(-h)=U^{T}(h)=Z(0)
$$

Taking (25) into account equation (21) takes a form

$$
U(0) A_{0}+Z(0) A_{1}+A_{0}^{T} U(0)+A_{1}^{T} Z^{T}(0)=-W .
$$

\section{A Lyapunov matrix for system with both lumped and distributed delay}

Matrices $U(\xi)$ and $Z(\xi)$ for time delay system with both lumped and distributed delay (1) which can be written in the form (6) have a form

$$
U(\xi)=\left[\begin{array}{ll}
U_{11}(\xi) & U_{12}(\xi) \\
U_{21}(\xi) & U_{22}(\xi)
\end{array}\right]
$$




$$
Z(\xi)=\left[\begin{array}{ll}
Z_{11}(\xi) & Z_{12}(\xi) \\
Z_{21}(\xi) & Z_{22}(\xi)
\end{array}\right]
$$

We take a matrix $W$ in a form

$$
W=\left[\begin{array}{cc}
W_{1} & 0 \\
0 & W_{2}
\end{array}\right]
$$

for $\xi \in[-h, 0]$, where $U_{11}(\xi), U_{12}(\xi), U_{21}(\xi), U_{22}(\xi), Z_{11}(\xi), Z_{12}(\xi), Z_{21}(\xi), Z_{22}(\xi)$, $W_{1}, W_{2}$ are $n \times n$ - real matrices. To obtain the Lyapunov matrix $U(\xi)$, given by formula (27), we should resolve the set of differential equations (24). Initial conditions of that differential equations fulfill the equality (26).

We write equations (24) and (26) for $A_{0}$ and $A_{1}$ given by (8) and (9)

$$
\begin{gathered}
\left(\begin{array}{l}
\frac{d U_{11}(\xi)}{d \xi}=U_{11}(\xi) A+U_{12}(\xi)+Z_{11}(\xi) B-Z_{12}(\xi) \\
\frac{d U_{1}(\xi)}{d \xi}=U_{21}(\xi) A+U_{22}(\xi)+Z_{21}(\xi) B-Z_{22}(\xi) \\
\frac{d U_{12}(\xi)}{d \xi}=U_{11}(\xi) C \\
\frac{d U_{22}(\xi)}{d \xi}=U_{21}(\xi) C \\
\frac{d Z_{11}(\xi)}{d \xi}=-B^{T} U_{11}(\xi)+U_{21}(\xi)-A^{T} Z_{11}(\xi)-Z_{21}(\xi) \\
\frac{d Z_{21}(\xi)}{d \xi}=-C^{T} Z_{11}(\xi) \\
\frac{d Z_{12}(\xi)}{d \xi}=-B^{T} U_{12}(\xi)+U_{22}(\xi)-A^{T} Z_{12}(\xi)-Z_{22}(\xi) \\
\frac{d Z_{22}(\xi)}{d \xi}=-C^{T} Z_{12}(\xi)
\end{array}\right. \\
\left\{\begin{array}{l}
U_{11}(0) A+A^{T} U_{11}(0)+U_{21}(0)+U_{12}(0)+Z_{11}(0) B+B^{T} Z_{11}(0)-Z_{12}(0)-Z_{12}^{T}(0)=-W_{1} \\
C^{T} U_{11}(0)+U_{21}(0) A+U_{22}(0)+Z_{21}(0) B-Z_{22}(0)=0 \\
U_{11}(0) C+A^{T} U_{12}(0)+U_{22}(0)+B^{T} Z_{21}^{T}(0)-Z_{22}(0)=0 \\
U_{21}(0) C+C^{T} U_{12}(0)=-W_{2}
\end{array}\right.
\end{gathered}
$$

Using the Kronecker product we can express (30) in a form

$$
\left\{\begin{array}{l}
\frac{d \operatorname{col}_{11}(\xi)}{d \xi}=\left(A^{T} \otimes I\right) \operatorname{col}_{11}(\xi)+\operatorname{col}_{12}(\xi)+\left(B^{T} \otimes I\right) \operatorname{colZ}_{11}(\xi)-\operatorname{colZ}_{12}(\xi) \\
\frac{d \operatorname{col}_{21}(\xi)}{d \xi}=\left(A^{T} \otimes I\right) \operatorname{col}_{21}(\xi)+\operatorname{col}_{22}(\xi)+\left(B^{T} \otimes I\right) \operatorname{col}_{21}(\xi)-\operatorname{colZ}_{22}(\xi) \\
\frac{d \operatorname{col}_{12}(\xi)}{d \xi}=\left(C^{T} \otimes I\right) \operatorname{col}_{11}(\xi) \\
\frac{d \operatorname{coll}_{22}(\xi)}{d \xi}=\left(C^{T} \otimes I\right) \operatorname{col}_{21}(\xi) \\
\frac{d \operatorname{col}_{11}(\xi)}{d \xi}=-\left(I \otimes B^{T}\right) \operatorname{col}_{11}(\xi)+\operatorname{col}_{21}(\xi)-\left(I \otimes A^{T}\right) \operatorname{colZ}_{11}(\xi)-\operatorname{colZ}_{21}(\xi) \\
\frac{d \operatorname{col} Z_{21}(\xi)}{d \xi}=-\left(I \otimes C^{T}\right) \operatorname{col}_{11}(\xi) \\
\frac{d \operatorname{col} Z_{12}(\xi)}{d \xi}=-\left(I \otimes B^{T}\right) \operatorname{col}_{12}(\xi)+\operatorname{col}_{22}(\xi)-\left(I \otimes A^{T}\right) \operatorname{col}_{12}(\xi)-\operatorname{col}_{22}(\xi) \\
\frac{d \operatorname{col} Z_{22}(\xi)}{d \xi}=-\left(I \otimes C^{T}\right) \operatorname{col}_{12}(\xi)
\end{array}\right.
$$


for $\xi \in[0, h]$ with initial conditions $\operatorname{colU}_{11}(0), \operatorname{col}_{21}(0), \operatorname{col}_{12}(0), \operatorname{col}_{22}(0)$, $\operatorname{colZ}_{11}(0), \operatorname{colZ}_{21}(0), \operatorname{col}_{12}(0), \operatorname{col}_{22}(0)$. Solution of the set of differential equations (32) is given in a form

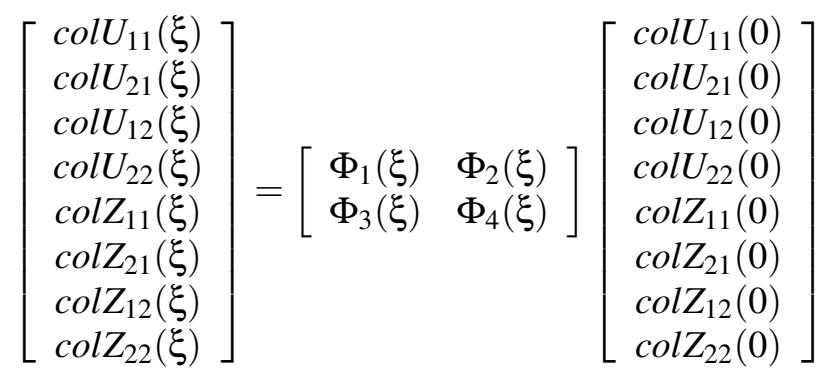

where a matrix $\Phi(\xi)=\left[\begin{array}{ll}\Phi_{1}(\xi) & \Phi_{2}(\xi) \\ \Phi_{3}(\xi) & \Phi_{4}(\xi)\end{array}\right]$ is a fundamental matrix of system (32). Equation (22) implies

$$
Z(h)=U(0) \text {. }
$$

It means

$$
\left[\begin{array}{l}
\operatorname{col}_{11}(h) \\
\operatorname{col}_{21}(h) \\
\operatorname{col} Z_{12}(h) \\
\operatorname{col} Z_{22}(h)
\end{array}\right]=\left[\begin{array}{l}
\operatorname{col} U_{11}(0) \\
\operatorname{col} U_{21}(0) \\
\operatorname{col} U_{12}(0) \\
\operatorname{col} U_{22}(0)
\end{array}\right]
$$

Equation (33) implies

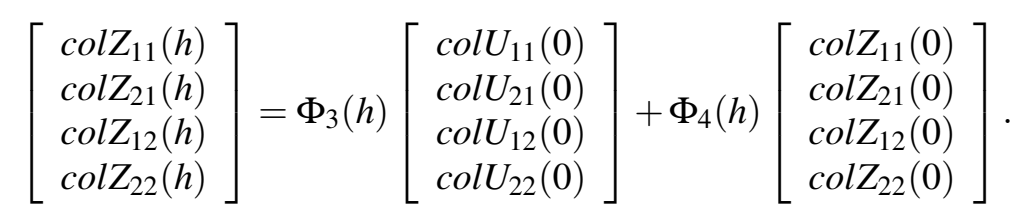

From equations (35) and (36) we obtain

$$
\left(\Phi_{3}(h)-I\right)\left[\begin{array}{l}
\operatorname{colU}_{11}(0) \\
\operatorname{colU}_{21}(0) \\
\operatorname{colU}_{12}(0) \\
\operatorname{colU}_{22}(0)
\end{array}\right]+\Phi_{4}(h)\left[\begin{array}{l}
\operatorname{colZ} \\
\operatorname{col}(112) \\
\operatorname{col}(0) \\
\operatorname{col}(2) \\
\operatorname{col}_{22}(0)
\end{array}\right]=0
$$


In this way we attain the set of algebraic equations which enables us to calculate the initial conditions of (32)

$$
\left\{\begin{array}{l}
U_{11}(0) A+A^{T} U_{11}(0)+U_{21}(0)+U_{12}(0)+Z_{11}(0) B+B^{T} Z_{11}(0)-Z_{12}(0)-Z_{12}^{T}(0)=-W_{1} \\
C^{T} U_{11}(0)+U_{21}(0) A+U_{22}(0)+Z_{21}(0) B-Z_{22}(0)=0 \\
U_{11}(0) C+A^{T} U_{12}(0)+U_{22}(0)+B^{T} Z_{21}^{T}(0)-Z_{22}(0)=0 \\
U_{21}(0) C+C^{T} U_{12}(0)=-W_{2} \\
\left(\Phi_{3}(h)-I\right)\left[\begin{array}{l}
\operatorname{col} U_{11}(0) \\
\operatorname{col} U_{21}(0) \\
\operatorname{col} U_{12}(0) \\
\operatorname{col}_{22}(0)
\end{array}\right]+\Phi_{4}(h)\left[\begin{array}{l}
\operatorname{col} Z_{11}(0) \\
\operatorname{colZ} 21(0) \\
\operatorname{col} Z_{12}(0) \\
\operatorname{col}\left(Z_{22}(0)\right.
\end{array}\right]=0
\end{array}\right.
$$

\section{Example}

Let us consider a system

$$
\left\{\begin{array}{l}
\frac{d x(t)}{d t}=a x(t)+b x(t-h)+\int_{-h}^{0} c x(t+\varsigma) d \varsigma \\
x(\theta)=\varphi(\theta)
\end{array}\right.
$$

for $t \geqslant 0, \theta \in[-2 h, 0]$, where $x(t) \in \mathbb{R}, a, b, c$ are real numbers, $h \geqslant 0$. The set of equations (32) can be written in a form

$$
\left[\begin{array}{c}
\frac{d U_{11}(\xi)}{d \xi} \\
\frac{d U_{21}(\xi)}{d \xi} \\
\frac{d U_{12}(\xi)}{d \xi} \\
\frac{d U_{22}(\xi)}{d \xi} \\
\frac{d Z_{11}(\xi)}{d \xi} \\
\frac{d Z_{21}(\xi)}{d \xi} \\
\frac{d Z_{12}(\xi)}{d \xi} \\
\frac{d Z_{22}(\xi)}{d \xi}
\end{array}\right]=\left[\begin{array}{cccccccc}
a & 0 & 1 & 0 & b & 0 & -1 & 0 \\
0 & a & 0 & 1 & 0 & b & 0 & -1 \\
c & 0 & 0 & 0 & 0 & 0 & 0 & 0 \\
0 & c & 0 & 0 & 0 & 0 & 0 & 0 \\
-b & 1 & 0 & 0 & -a & -1 & 0 & 0 \\
0 & 0 & 0 & 0 & -c & 0 & 0 & 0 \\
0 & 0 & -b & 1 & 0 & 0 & -a & -1 \\
0 & 0 & 0 & 0 & 0 & 0 & -c & 0
\end{array}\right]\left[\begin{array}{c}
U_{11}(\xi) \\
U_{21}(\xi) \\
U_{12}(\xi) \\
U_{22}(\xi) \\
Z_{11}(\xi) \\
Z_{21}(\xi) \\
Z_{12}(\xi) \\
Z_{22}(\xi)
\end{array}\right]
$$


A fundamental matrix of system (40) has a form $\Phi(\xi)=\left[\Phi_{i j}(\xi)\right]$ for $i, j=1, \ldots, 8$ The set of algebraic equations (38) is given by formula

$\left[\begin{array}{cccccccc}2 a & 1 & 1 & 0 & 2 b & 0 & -2 & 0 \\ c & a & 0 & 1 & 0 & b & 0 & -1 \\ c & 0 & a & 1 & 0 & b & 0 & -1 \\ 0 & c & c & 0 & 0 & 0 & 0 & 0 \\ p_{51}-1 & p_{52} & p_{53} & p_{54} & p_{55} & p_{56} & p_{57} & p_{58} \\ p_{61} & p_{62}-1 & p_{63} & p_{64} & p_{65} & p_{66} & p_{67} & p_{68} \\ p_{71} & p_{72} & p_{73}-1 & p_{74} & p_{75} & p_{76} & p_{77} & p_{78} \\ p_{81} & p_{82} & p_{83} & p_{84}-1 & p_{85} & p_{86} & p_{87} & p_{88}\end{array}\right]\left[\begin{array}{c}U_{11}(0) \\ U_{21}(0) \\ U_{12}(0) \\ U_{22}(0) \\ Z_{11}(0) \\ Z_{21}(0) \\ Z_{12}(0) \\ Z_{22}(0)\end{array}\right]=\left[\begin{array}{c}-w_{1} \\ 0 \\ 0 \\ -w_{2} \\ 0 \\ 0 \\ 0 \\ 0\end{array}\right]$

where $p_{i j}=\Phi_{i j}(h), w_{1}, w_{2}$ are positive real numbers.

Figures show the elements of the Lyapunov matrix $U(\xi)$ for given values of the system (39) parameters. Figure 1 shows the elements of the Lyapunov matrix $U(\xi)$ for $a=-0.2, b=-1, c=1.2, h=1$ and Figure 2 for $a=-0.4, b=-1, c=1.4, h=1$.

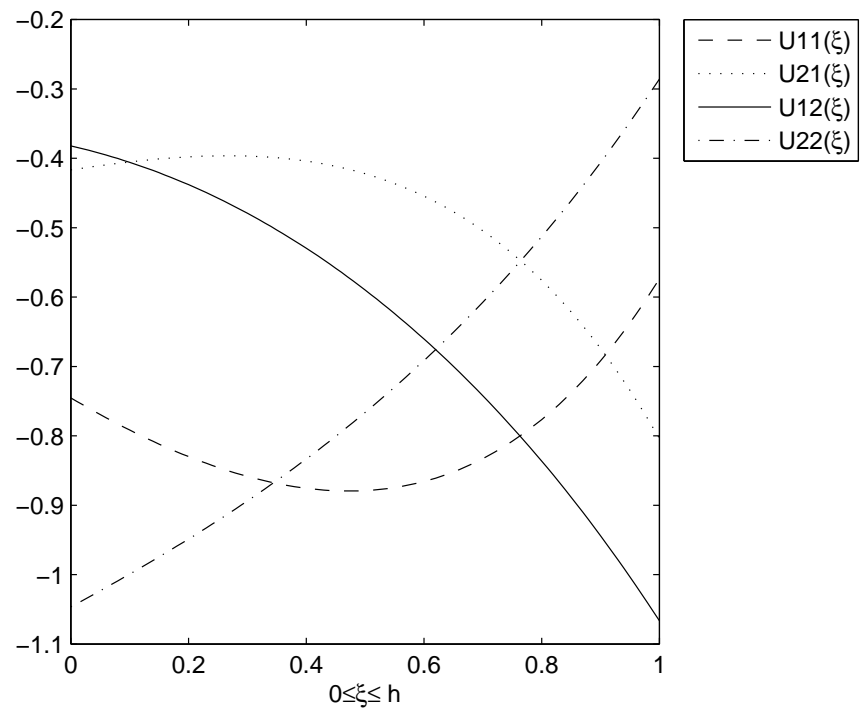

Figure 1: Elements of the Lyapunov matrix for $\mathrm{a}=-0.2, \mathrm{~b}=-1, \mathrm{c}=1.2, \mathrm{~h}=1$ 


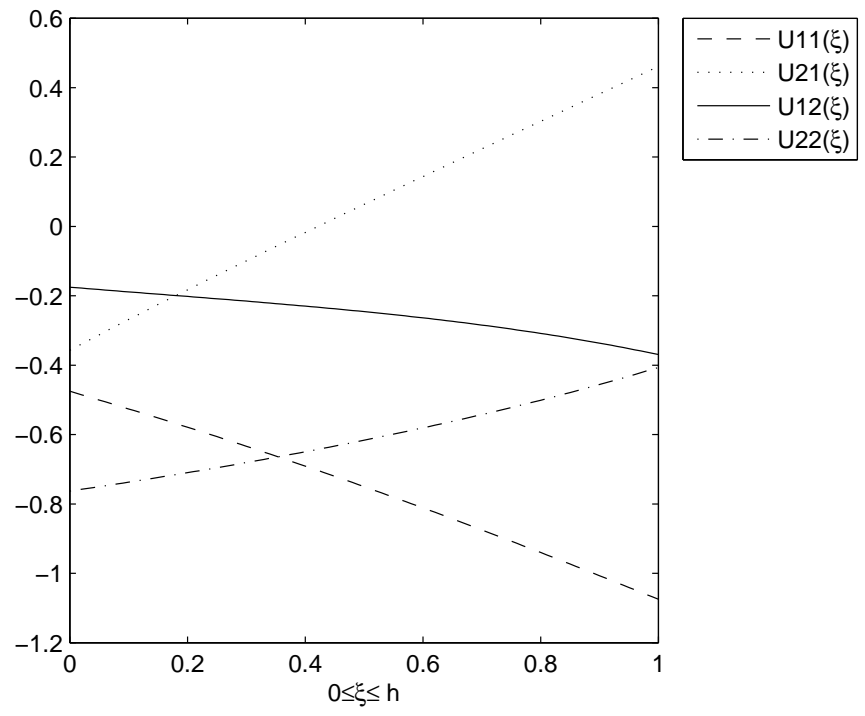

Figure 2: Elements of the Lyapunov matrix for $a=-0.4, b=-1, c=1.4, h=1$

\section{Conclusions}

In the paper a Lyapunov functional for time delay system with both lumped and distributed time delay is presented. The Lyapunov functional is determined by means of the Lyapunov matrix. The method of determination of the Lyapunov matrix for time delay system with both lumped and distributed delay is also presented. The Lyapunov functional has the property that its time derivative computed along solution of the system is equal to the negative definite quadratic form of the instant state of the dynamical system with time delay. An example, illustrating the method, is also given.

\section{References}

[1] R. Bellman, and K. Cooke: Differential-difference Equations. New York, Academic Press, 1963.

[2] S. BiAŁAS and H. GóRECKI: Generalization of Vieta's formulae to the fractional polynomials, and generalizations the method of Graeffe-Lobachevsky. Bulletin of the Polish Academy of Sciences Technical Sciences, 58 (2010), 625-629.

[3] J. Duda: Lyapunov functional for a linear system with two delays. Control and Cybernetics, 39 (2010), 797-809. 
[4] J. DUDA: Lyapunov functional for a system with k-non-commensurate neutral time delays. Control and Cybernetics, 39 (2010), 1173-1184.

[5] J. DUDA: A Lyapunov functional for a neutral system with a time-varying delay. Bulletin of the Polish Academy of Sciences Technical Sciences, 61 (2013), 911-918.

[6] J. DUDA: Lyapunov matrices approach to the parametric optimization of timedelay systems. Archives of Control Sciences, 25 (2015), 279-288.

[7] J. DUDA: Lyapunov matrices approach to the parametric optimization of a neutral system. Archives of Control Sciences, 26 (2016), 81-93.

[8] J. DUDA: Lyapunov matrices approach to the parametric optimization of a system with two delays. Archives of Control Sciences, 26 (2016), 281-295.

[9] J. DUDA: A Lyapunov functional for a neutral system with a distributed time delay. Mathematics and Computers in Simulation, 119 (2016), 171-181.

[10] J. DUDA: Lyapunov matrices approach to the parametric optimization of a neutral system with two delays. Mathematics and Computers in Simulation, 136 (2017), 22-35.

[11] E. FRIDMAN: New Lyapunov-Krasovskii functionals for stability of linear retarded and neutral type systems. Systems \& Control Letters, 43 (2001), 309-319.

[12] H.GóRECKI and S.BiAŁAS: Relations between roots and coefficients of the transcendental equations. Bulletin of the Polish Academy of Sciences Technical Sciences, 58 (2010), 631-634.

[13] H. Górecki, S. Fuksa, P. Grabowski and A. Korytowski: Analysis and Synthesis of Time Delay Systems. John Wiley \& Sons. Chichester, New York, Brisbane, Toronto, Singapore, 1989.

[14] H. GóreCKI and L. POPEK: Parametric optimization problem for control systems with time-delay. 9th World Congress of IFAC IX, CD-ROM, (1984).

[15] K. GU: Discretized LMI set in the Stability Problem of Linear Time Delay Systems. Int. J. of Control, 68 (1997), 923-934.

[16] K. GU and Y. LIU: Lyapunov-Krasovskii functional for uniform stability of coupled differential-functional equations. Automatica, 45 (2009), 798-804.

[17] Q.L. HAN: On robust stability of neutral systems with time-varying discrete delay and norm-bounded uncertainty. Automatica, 40 (2004), 1087-1092.

[18] Q.L. HAN: A descriptor system approach to robust stability of uncertain neutral systems with discrete and distributed delays. Automatica, 40 (2004), 1791-1796. 
[19] Q.L. HAN: On stability of linear neutral systems with mixed time delays: A discretised Lyapunov functional approach. Automatica 41 (2005), 1209-1218.

[20] Q.L. HAN: A new delay-dependent stability criterion for linear neutral systems with norm-bounded uncertainties in all system matrices. Int. J. of Systems Science, 36 (2005), 469-475.

[21] Q.L. HAN: A discrete delay decomposition approach to stability of linear retarded and neutral systems. Automatica, 45 (2009), 517-524.

[22] Q.L. HAN: Improved stability criteria and controller design for linear neutral systems. Automatica, 45 (2009), 1948-1952.

[23] D. Ivanescu, S.I. Niculescu, L. Dugard, J.M. Dion and E.I. Verriest: On delay-dependent stability for linear neutral systems. Automatica, 39 (2003), 255-261.

[24] V.L. KHARITONOV: Lyapunov functionals and Lyapunov matrices for neutral type time delay systems: a single delay case. Int. J. of Control, 78 (2005), 783-800.

[25] V.L. Kharitonov: Lyapunov matrices for a class of time delay systems. Systems \& Control Letters, 55 (2006), 610-617.

[26] V.L. KHARITONOV: Lyapunov matrices for a class of neutral type time delay systems. Int. J. of Control, 81 (2008), 883-893.

[27] V.L. KhaRitonov: On the uniqueness of Lyapunov matrices for a time-delay system. Systems \& Control Letters, 61 (2012), 397-402.

[28] V.L. Kharitonov and D. HinRichsen: Exponential estimates for time delay systems. Systems \& Control Letters, 53 (2004), 395-405.

[29] V.L. KhARITONOV and E. PlisChKE: Lyapunov matrices for time-delay systems. Systems \& Control Letters, 55 (2006), 697-706.

[30] V.L. KhaRitonov and A.P. ZhABKo: Lyapunov-Krasovskii approach to the robust stability analysis of time-delay systems. Automatica, 39 (2003), 15-20.

[31] J. Klamka: Controllability of Dynamical Systems. Kluwer Academic Publishers Dordrecht, 1991.

[32] YU and M. RePIN: Quadratic Lyapunov functionals for systems with delay. Prikl. Mat. Mekh., 29 (1965), 564-566. 